\title{
013Novel Method of Substitution for Combine Through-and-through Angle of the Mouth, Fragments of Upper and Lower Lips Defect
}

\author{
Igor Zaderenko, 2, *, Ali Mudunov', , , Sergey Ivanov², Sevil Alieva', Vasiliy Tsiklauri', \\ Alexander Kazimov', David Safarovi, Alexandra Eremina ${ }^{2}$, Alena Hromushina ${ }^{2}$, \\ Bulat Bektimirov', Alexander Dymnikov ${ }^{2}$ \\ ${ }^{1}$ Department of Oncology and Surgical Treatment (Head and Neck Tumors), Federal State Budgetary Institution «N. N. Blokhin National \\ Medical and Research Center of Oncology» of the Ministry of Healthcare of the Russian Federation, Moscow, Russia \\ ${ }^{2}$ Department of Maxillo-facial Surgery and Operative Dentistry, Peoples Friendship University of Russia, Moscow, Russia
}

\section{Email address:}

Igorakis@list.ru (I.Zaderenko), ali.mudunov@inbox.ru (A. Mudunov), syivanov@yandex.ru (S. Ivanov), drsevil@mail.ru (S. Alieva), vasya84@rambler.ru(V.Tsiklauri),mr.kazimov@yandex.ru(A. Kazimov), safarowd@mail.ru (D. Safarov), sas1.91@mail.ru(A. Eremina), alenka-khromushina@yandex.ru (A. Hromushina),bulat.temir@bk.ru (B. Bektimirov), al.dymnikov@gmail.com (A. Dymnikov)

${ }^{*}$ Corresponding author

\section{To cite this article:}

Igor Zaderenko Ali Mudunov, Sergey Ivanov, Sevil Alieva, Vasiliy Tsiklauri, Alexander Kazimov, David Safarov, Alexandra Eremina, Alena Hromushina, Bulat Bektimirov, Alexander Dymnikov. 013Novel Method of Substitution for Combine Through-and-through Angle of the Mouth, Fragments of Upper and Lower Lips Defect. Journal of Surgery. Vol. 8, No. 1, 2020, pp. 22-27. doi: 10.11648/j.js.20200801.15

Received: December 5, 2019; Accepted: January 29, 2020; Published: February 10, 2020

\begin{abstract}
Through-and-through defect of soft tissues of the cheek, mouth angles, upper and lower lip originate from surgical intervention of tumors of the oral cavity involving external skin, soft tissue and oral lining. The article describes an original method of one-stage reconstruction in N. N. Blokhin National Medical and Research Center of Oncology. Russian Federation's patent № 2489096 was granted for this method. We propose to use 3 different flaps from different anatomic areas, which have varying histological structures and independent blood supply. The first flap - muscle, is formed from the biggest part of the muscles masseter from the affected side and serves to restore the functions of the lips and cheek due to the fact that after the formation of the flap two its parts fixed to the remnants of the muscles of the upper and lower lips and part of the buccal muscle. The flap is perfusion by a. maxillaris and a. facialis. The second flap - musculocutaneous, formed from the skin of areas adjacent to the defect, nasolabial or submandibular. The flap serves to replace a skin defect, defect of the cheek, upper and lower lips. The flap is perfusion by a. facialis. The third flap - fascial, formed from the temporal fascia on the affected side. The temporal fascial flap is used to replace the defect of the mucous membrane of the cheek, upper and lower lips, in addition, the red border of the lips and the corner of the mouth. The flap is perfusion by aa. temporalis profunda et superficialis. All three flaps perfectly carry out hermetic sealing of the oral cavity, independent perfusion of each flap, even in the case of necrosis of one of the flaps, allows preservation of hermeticism. The use of three flaps with different histological structure allows to achieve a good functional and aesthetic result. Flaps perform various functions, ensuring the functional and anatomical usefulness of the resected tissues. By improving the blood suppling of flaps, we reduce time for patient's rehabilitation and postoperative damage.
\end{abstract}

Keywords: Plastic, Replacement, Defect, Reconstruction, Flap

\section{Introduction}

The need for replacement such extensive through-andthrough combined defects of the cheek, mouth corner, part of the upper and lower lips does not occur often, but it is a serious problem of patient's rehabilitation. The etiology of such defects is variable: malignant neoplasms, operations, radiation therapy, various mechanical, chemical, thermal injuries, complications of specific infections [1, 2, 8-10]. 
Substitution of such composite defects has particular qualities: 1. It is necessary to create an internal lining, 2. Necessity of doubling plastic material for recovery of organ functional activity, 3. Importance this zone of aesthetic perception of a person [3- $6,11-15]$.

\section{Materials and Methods}

The maxillofacial region includes tissues that are different in histological affiliation and function. Replacing defects in this area involves the reconstruction of bone, muscle, skin and mucous membrane. These tissues are a complex anatomical and functional unit, to what simultaneous reconstruction is necessary for fully restoring lost functions. Through-and-through may lead to violation of hermeticity of the oral cavity and as a result - functional problems with eating and swallowing, chewing and speech. In additionconstant salivation, bad breath, infection and maceration of tissues around the defect. Such an extensive problems causes severe physical suffering, which leads patient to social maladaptation and disability. Here are methods for replacing such defects.

A well-known reconstruction method for through-andthrough defects of the maxillofacial region is usage a free thoracodorsal flap [1]. A bordering defect incision is made to mobilize its edges. At the beginning make incision (approximately $5 \mathrm{~cm}$ ) on the side of the defect in the submandibular region, skin, subcutaneous fat, muscle are dissected, facial vessels isolated. Formed subcutaneous tunnel between the lower edge of the defect and the wound (for holding the vascular pedicle), $3-4 \mathrm{~cm}$ wide. While patient is lying on his side with a raised and fixed upper limb, flap contours are applying to the axillary skin according to the shape and size of the defect. When creating the inner lining with flap tissues, the contours must extend it by the amount of the defect. The contours of the flap are applied so that the edge of the flap is located in the projection of the front edge of the muscle's latissimus dorsi. Flap is formed, highlighting the neurovascular bundle of the flap all the way to its entry into the muscle. Making sure that the upper horizontal border of the flap passes above the level of entry of the vascular bundle into the muscle. Cross the vascular pedicle of the flap. Stitched the central ends of the vessels. The wound is sutured after a wide mobilization of the edges. Then the flap moved to the defect so that the entry of vascular pedicle into the flap matches to the lower border of the defect. The skin area of these flap is placed in the direction of the defect, to create the inner lining and fixed to the edges of the defect with several stitches-holders. The vascular pedicle of the flap passed through the tunnel into the wound in the submandibular region to the recipient vessels. Stitch the flap vessels and the facial vessels end to end. After blood flow restoration the flap fixed to the inner edges of the defect, de-epidermis it in the inflection point and complete its fixation to the outer edges. This method gives a good aesthetic and functional result. However, plastic surgery using microvascular anastomoses is technically difficult. In addition, microsurgical surgery is possible only in the presence of intact recipient vessels and a good somatic condition of patients. Blood supply is carried out due to one main vessel, which is stitched with the recipient vessel.

The next method for replacing defects of the oral cavity with organotypic plastic material was developed at the Department of Maxillofacial Surgery in Belarusian Medical University [3]. At the first stage forms organotypic plastic material. For this, cut out a musculocutaneous tongue-like flap on the feeding pediculus with inclusion nearby facial muscles, which are fixed to the distal part of the flap. With electrodermatome from the inner surface of the shoulder takes a split flap 0.2-0.4 mm thickness. Autodermoflaps placed on the wound surface of the tongue-like flap sutured to its edges. Fixing the flap in the same position creates optimal tissue conditions that ensure complete engraftment of the autoderm flap as a muscle component of organotypic plastic material. After 14-18 days, you can perform plastic reconstruction of through defects. At the second stage of treatment, removing tumor of the oral area with a simultaneous compensation with organotypic plastic material (OPM). A skin incision is made along the line of the previous scar with the release of the OPM, and then the OPM is modeled according to the shape and true dimensions of the primary defect. The OPM moved to the area of the defect and stitched to its edges in a state of physiological tension. The edges of the mucosal defect are connected with sutures, the muscle layer of the OPM with the remainder of the circular muscle of the mouth in the area of the defect, the skin part of the OPM with the red border of the lips or skin edges of the defect.

The autoflap in the area of the mother bed excised, and the wound is sutured. If during excision of the autoflap the size of the resulting defect does not allow it to be covered with local tissues, the autoflap can be left. The disadvantages of this method are: multi-stage, additional trauma to surrounding healthy tissues and donor wound, limited use of this method for malignant tumors due to the duration of formation and replacement of flap. Blood supplying to the flap occurs due to the vessels passing in the pediculus of the flap.

The third way to replace defects of the maxillofacial region - with flap on the feeding pediculus and forming lining from a free skin flap [7]. In the area of the nasolabial fold (if it is necessary to eliminate the defect of the upper lip), the chin (the defect of the lower lip), the upper part of the front surface of the chest (defects of the lips, corners of the mouth, cheeks) cut out a tongue-like or bridge-like skin flap of a certain thickness up to $1 \mathrm{~cm}$. The wound surface of the flap and the mother's bed are closed with a free autodermal split flap, which is usually taken from the inner surface of the shoulder or front surface of the thigh with a thickness of $0.35 \mathrm{~mm}$. This duplicated flap place in wound and sutured to the edges. This ensures immersion of the split graft under the skin for its engraftment. The average immersion time for a split skin graft is 19.8 days. After 18-20 days, the formed epithelized flap with a well-established split 
dermatoflap on the other hand is again cut out and moved directly to the edge of the defect, where it is sutured with three-layer sutures: the edges of the mucous membrane defect - with a split graft from the side of the epithelized flap, the edges of the muscle layer - with subcutaneous tissue flap, skin edges of the defect with flap skin. If the flap was cut out near the defect plastic consisted of two stages and took on average about a month; when cutting flap was far away from the defect, an additional stage of flap migration was introduced, and the duration of plastic surgery increased to 1.5-2 months. The disadvantages of this method are the time duration: the treatment is carried out in several stages and takes total about 1.5-2 months, which for patients with cancer leads for delaying surgery for the tumor.

Another way to replace the maxillofacial region is to cut two musculocutaneous flaps on the feeding pediculus in the area adjacent to the postoperative defect. The size of the flaps to be cut exceeds the defect size by $30-50 \%$. In this case, the edge of the first flap is sewn to the edge of the second flap. On their adipose tissue or periosteum transplanted a free musculocutaneous autograft taken from the inner surface, for example, the auricle or the inner surface of the shoulder. The dimensions of the graft take more than a defect, taking into account about $30 \%-50 \%$ of it reduction, but accordingly the wound surface of the flap, which will cover the internal defect of the lip, cheek, corner of the mouth, nasal cavity of the wing of the nose, the outer wall of the maxillary sinus. The flap is pressed with gauze turundas with iodoform in order to prevent hematoma and autograft necrosis [4]. The disadvantages of this method are: additional serious injury to surrounding tissues (the formation of a musculocutaneousperiosteal flap), with function violation of facial muscles in displaced flap and donor site, one feeding pediculus of the flap.

The experience of many maxillofacial clinics has convincingly proven how necessary primary plastic surgery is. Subsequently, due to ongoing scarring and tissue movement, conditions for plastic surgery become significantly worse. Currently, it is proved that the initial reconstructive operations do not contribute to the occurrence of tumors relapse. Moreover, the percentage of relapses in primary repair is significantly lower than in delayed operations. In particular, relapses develop only along the edge of the skin graft, not growing into it. Therefore, an increasing number of surgeons are inclined to think about the need of simultaneously perform plastic surgery to remove the resulting defects to remove the tumor. In this regard, we have developed an original method for replacing the through defect of the neck, angle of the mouth, upper and lower lips.

Our method performed under endotracheal anesthesia. After defect formation, the circular muscle of the mouth and the remaining parts of the upper and lower lips are simultaneously mobilized. Mobilization is carried out approximately to the middle line. We formed three different flaps with independent blood supplying, different histological structure and performing different functions (Figures 1, 2, 3).

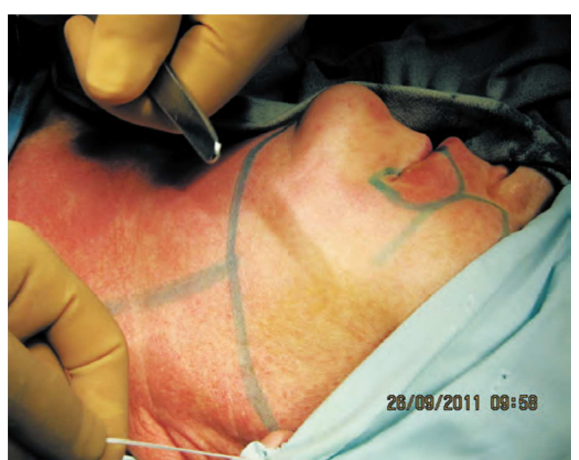

Figure 1. Tumor of the buccal mucosa, extending to the skin.

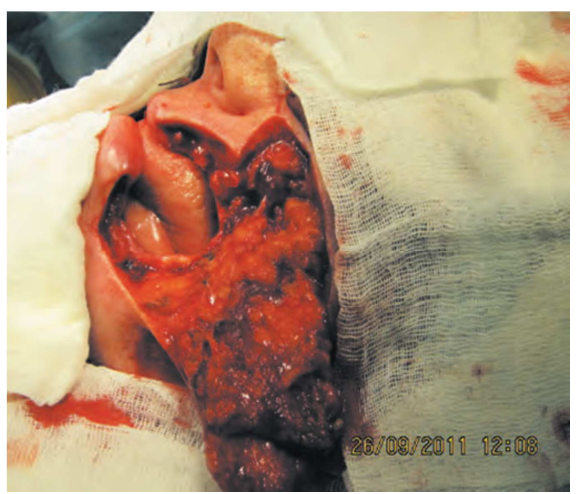

Figure 2. Formed defect (side view).

The first flap is formed from $2 / 3$ of the chewing muscle on the same side. It restored the function of the circular muscle of the mouth. The nutrition of the flap is carried out by the chewing artery. Made incision is submandibular region from the same side. The chewing muscle is segregate from the place of attachment to the angle of the jaw to fixation to the zygomatic bone. The chewing muscle separated from the place of attachment to the lower jaw. From $2 / 3$ of the chewing muscle we formed a muscle flap, which is divided into 2 equal parts (legs). The mobility of the flap should be sufficient, so that the fixation to the fragments of the upper and lower lips occurs without pronounced tension. Further, in the soft tissues of the preserved part of the cheek create a tunnel to the defect of the corner of the mouth, upper and lower lips (Figure 4).

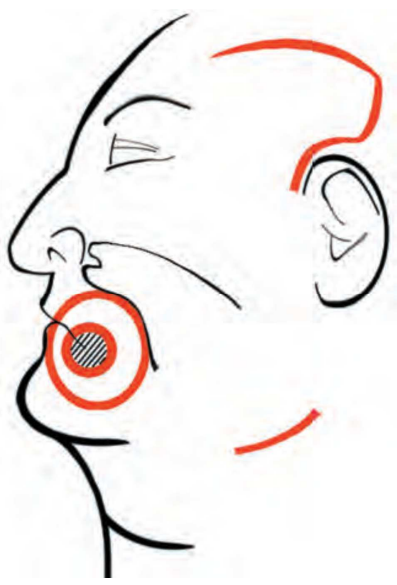

Figure 3. Scheme of the cutting lines. 


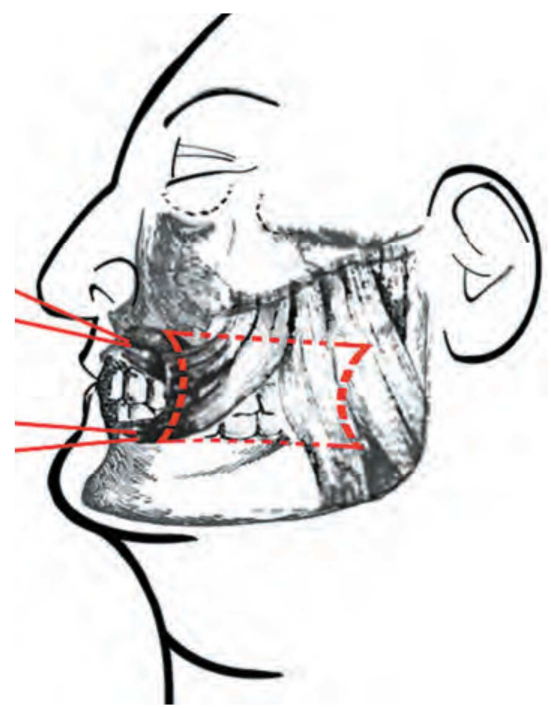

Figure 4. Flap formation from chewing muscle.

The muscle flap is carried through the formed tunnel to the corner of the mouth and the remaining parts of the upper and lower lips. Each pediculus of the formed muscle flap are fixed to the remains part of the circular muscle of the mouth and upper and lower lips by separate U-shaped double-row sutures (Figure 5).

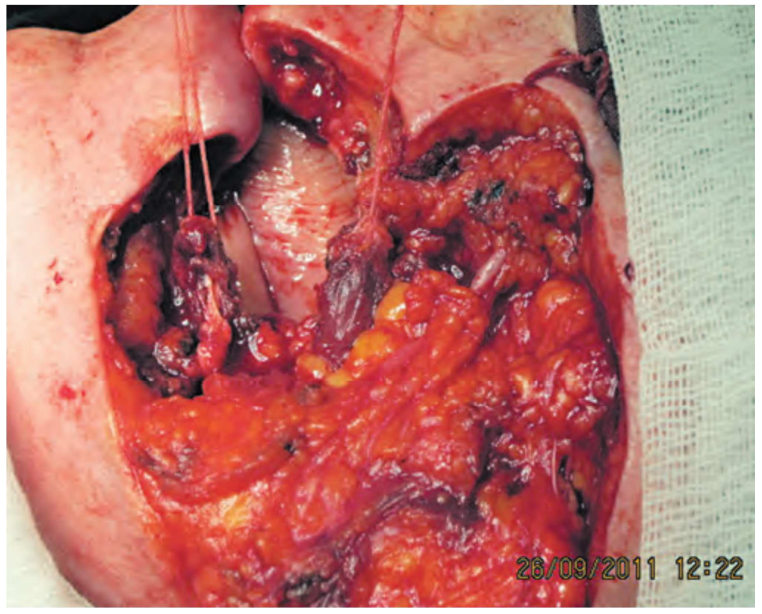

Figure 5. The formed flap of the chewing muscle fixed to the remains of the circular muscle.

The second flap is rotational, musculocutaneous, from adjacent anatomical regions, nasolabial or submandibular. The choice of the donor site in this case depends on the condition of the skin, size of the defect and localization in relation to the corner of the mouth, which replaces the skin defect of the upper and lower lips, cheeks, mouth angle. The nutrition of the flap is carried out by the facial artery. We formed the rotational musculocutaneous flap from neighboring anatomical regions (submandibular or nasolabial) with the base facing the branch of the jaw. Size of the flap must match the formed defect. At the top of the flap, it is necessary to cut out a triangular skin area (from all layers of flap), in the form of a "dovetail", which is necessary for the subsequent formation of the mouth corner. The previously formed rotational musculocutaneous flap moves to the defect and fixed by separate nodular sutures.

The third flap is the temporal fascial flap, replacing the defect of the mucous membrane, the defect of the red border of the upper, lower lips and the corner of the mouth. The nutrition and blood supply of this flap comes from the superficial temporal artery.

In the temporal region, a temporal fascial flap is cut out according to the standard technique. It should be noted that this flap should also be as mobile as possible. A tunnel is formed under the zygomatic arch into the oral cavity (Figure $6)$. Through the formed tunnel, the temporal flap is removed into the oral cavity. The temporal flap is fixed by separate nodular sutures to the defect of the mucous membrane of the cheek, lips, legs of the formed flap of the chewing muscle. The wound of the temporal-parietal region is sutured with separate nodular sutures. Part of the flap is turned out and sewn to the displaced musculocutaneous flap, thereby forming a red border and the corner of the mouth (Figure 7).

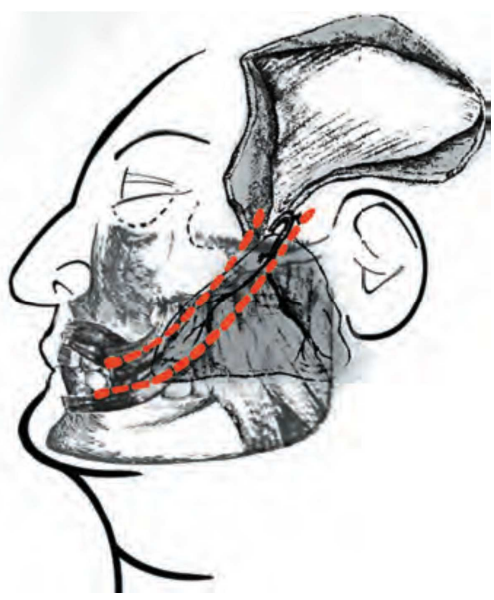

Figure 6. Formation of fascial- temporal flap.

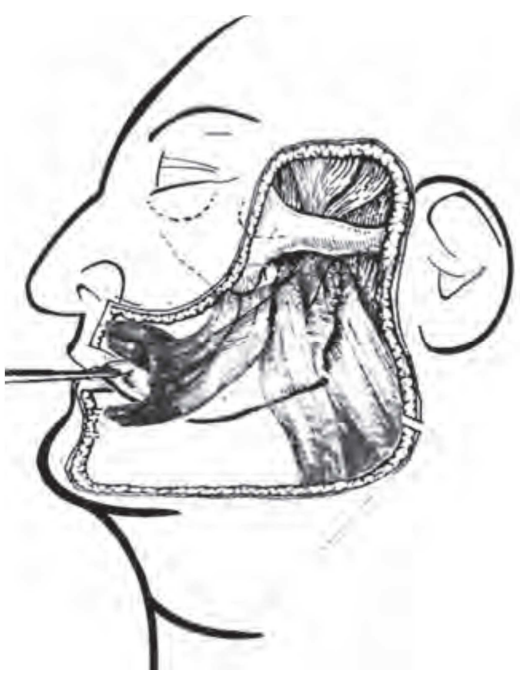

Figure 7. Replacement of the cheeks mucosal defect, formation angle of the mouth and the red border lips.

The final step is to suture the donor wound at the site of the musculocutaneous flap (Figures 8, 9). 


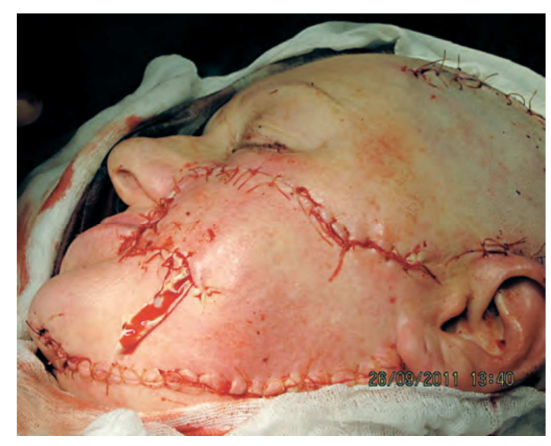

Figure 8. Postoperative seams.

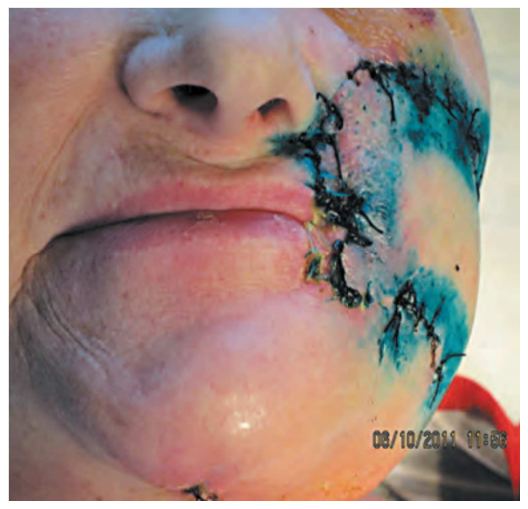

Figure 9. Ninth day.

The advantages of the proposed method are - one-stage repair and simplicity of execution. This kind of original method of reconstruction of extensive through-and-through defect soft tissues of the cheek, the corner of the mouth, fragments of the upper and lower lips provides a good functional and cosmetic result; sealing the oral cavity, free movements of the lips, normal eating and speech. In this regard using our method we improve quality of life, psychological and social rehabilitation. Having an independent blood supplying, each of the flaps remains viable in violation of trophic. The method does not require additional surgical correction. Due to the good nutrition of the flaps, it is possible to conduct radiation therapy in the postoperative period in radical doses with minimal postradiation toxicity, even in the case of necrosis one of the flaps (Figures 10, 11).

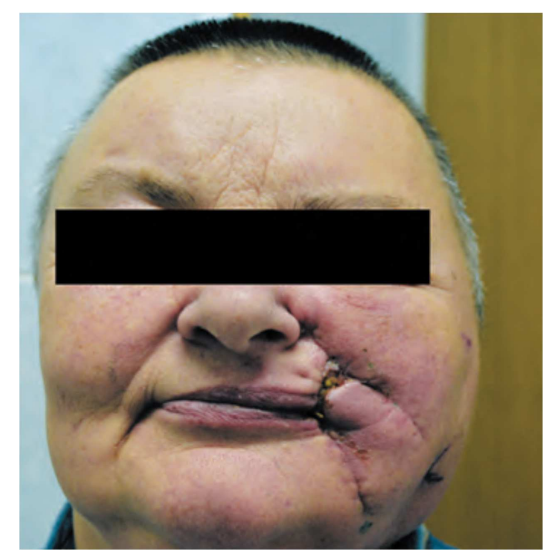

Figure 10. Partial necrosis of the skin flap.

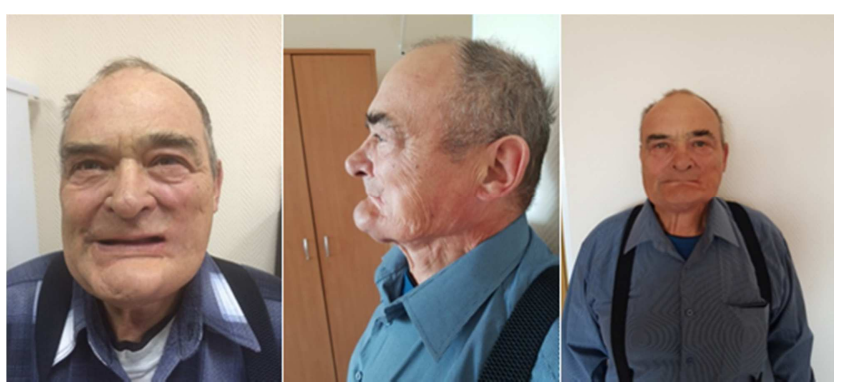

Figure 11. Three years after treatment, without local and regional relapse. Functional result is good.

\section{Conclusion}

The novel method of substitution for combine throughand-through defect angle of the mouth, fragments of upper and lower lips have many advantages. This technique allows one-stage repair, optimal restoration of function, anatomical shape, elimination of cosmetic defect by using 3 flaps with independent blood supply, histological structure and purpose. Tissues of the donor zone of the musculocutaneous flap are identical in color and structure. The repair is simple to perform and does not require the involvement of expensive specialists and equipment (for example, microsurgical). The use of this technique can reduce the duration of operation and less correction with reduction duration of treatment and hospital stay, significantly reduces the incidence of complications. This technique allows for early full rehabilitation of patients. If necessary, it is possible to conduct additional radiation therapy in radical doses early after surgery.

\section{References}

[1] Nerobeev A., Plotnikova N. Reconstructive surgery of the maxillofacial soft tissue region: A guidelines. Moscow: Medicine, 1997.

[2] Bernadsky Yu. I. Traumatology and reconstructive surgery of the craniofacial region. M: Medical literature, 2006.

[3] Chudakov O. P., Trizna N. M., Tesevich L. I. Options for compensating through defects in the quantum region using organotypic plastic material. Patent № a20040633. 07.07.2004.

[4] Durnovo E. A., Mishina N. V., Khomutinnikova N. E., et al. Plastic surgery of through-and-through defects of the maxillofacial region patients with cancer. Modern technologies in medicine 2010; 4: 86-91.

[5] Perlukhin M. L., Sulimov A. F., Akhmetyanov A. Sh. Elimination of defects in the maxillofacial region by using microsurgical autotransplants. Siberian journal of Oncology 2008; 1 (25): 106-7.

[6] Weerda H. Reconstructive facial plastic surgery. A problemsolving manual. Stuttgart - New-York, 2001. P. 4-6.

[7] Косметические операции лица /Под ред. Михельсона Н. М. - М., Медицина, 1962, С. 123-124. 
[8] Wu, J. C.-H., Lee, Y.-C., Cheng, Y.-C., \& Wu, C.-W. (2017). Reconstruction of Through-and-through Oromandibular Defect. Plastic and Reconstructive Surgery - Global Open, 5 (2), e1212.

[9] Jeng, S.-F., Kuo, Y.-R., Wei, F.-C., Su, C.-Y., \& Chien, C.-Y. (2004). Reconstruction of Concomitant Lip and Cheek Through-and-Through Defects with Combined Free Flap and an Advancement Flap from the Remaining Lip. Plastic and Reconstructive Surgery, 113 (2), 491-498.

[10] Karapandzic, M. Reconstruction of lip defects by local arterial flaps. Br. J. Plast. Surg. 47: 422, 1994.

[11] Kroll, S. S. Staged sequential flap reconstruction for large lower lip defects. Plast. Reconstr. Surg. 88: 620, 1991.

[12] Liu WC, Yang KC. One-stage through-and-through cheek, lips, and oral commissure reconstruction using a double- paddle peroneal chimeric flap: an innovative method. Head Neck. 2015; 37 (5): 662-9.

[13] Ethunandan M, Macpherson DW, Santhanam V. Karapandzic flap for reconstruction of lip defects. J Oral Maxillofac Surg. 2007; 65 (12): 2512-7.

[14] Yamauchi M, Yotsuyanagi T, Ezoe K, Saito T, Yokoi K, Urushidate S. Estlander flap combined with an extended upper lip flap technique for large defects of lower lip with oral commissure. J Plast Reconstr Aesthet Surg. 2009; 62 (8): 9971003.

[15] Sakakibara A, Matsumoto K, Hasegawa T, Minamikawa T, Komori T. Single-stage reconstruction for buccal mucosa tumor resection including the labial commissure using a facial artery musculomucosal flap and a vermilion advancement flap. J Surg Case Rep. 2017; 20 (6): rjx108. 